\title{
Genetics
}

\section{COMPREHENSIVE FISH TESTING USING FFPE TISSUE MICROARRAY TO SCREEN FOR SECONDARY ABNORMALITIES IN MANTLE CELL LYMPHOMA: A RETROSPECTIVE STUDY}

Fiona Webb $^{1}$, Collette Mahler-Hindson ${ }^{2}$, Monica Armstrong ${ }^{1}$, Jacqueline Cosh ${ }^{1}$, Amanda Bullman ${ }^{1}$, RayMun Koo ${ }^{3}$, Nalini Pati ${ }^{3}$, Adrienne Morey ${ }^{1,2,4}$, Dipti Talaulikar ${ }^{1,2,3}$ ${ }^{1}$ Department of Diagnostic Genomics, ACT Pathology, Canberra, Australia; ${ }^{2}$ Australian National University, Canberra, Australia; ${ }^{3}$ Department of Haematology, ACT Pathology, Canberra, Australia; and ${ }^{4}$ Department of Anatomical Pathology, ACT Pathology, Canberra, Australia

Aim: Mantle cell lymphoma (MCL), is characterised by the $t(11 ; 14)$. Detection of $t(11 ; 14)$ by FISH is used in diagnosis. Clinically significance of additional abnormalities remains unclear. Our aim was to use a comprehensive FISH panel to identify secondary changes in a retrospective cohort of MCL samples.

Method: FISH was performed retrospectively on 28 FFPE lymph node samples from patients diagnosed with MCL. Lymph node punch biopsies were constructed into a tissue microarray of 10 samples per slide in duplicate. The FISH probe panel was designed to detect rearrangements of BCL6, MYC and BCL2 and copy number loss of ATM and TP53 and confirmation of the presence of the $\mathrm{t}(11 ; 14)$.

Result: At least one additional abnormality including losses of TP53 (5/26), ATM (11/26), polysomies for MYC (8/26), BCL6 $(18 / 26)$ and BCL2 (10/26) and rearrangements of MYC (2/26) and BCL6 (1/26) was observed in $24 / 28(86 \%)$ cases. Two or more additional changes were observed in 13/28 (46\%) patients. One patient had triple-hit with rearrangements of CCND1, MYC and BCL6.

Conclusion: Expansion of the FISH probe panel detected additional abnormalities in $26 / 30(86 \%)$ of patients including a triple hit case.

\section{HERITABLE CANCER RISK IN THE GENOMIC ERA}

Mandy L. Ballinger $^{1,2}$, David Thomas ${ }^{1,2}$

${ }^{1}$ Garvan Institute of Medical Research, Sydney, NSW, Australia; and ${ }^{2}$ St Vincent's Clinical School, University of NSW, NSW, Australia

Traditional family history based identification of heritable cancer predisposition is giving way to genomic technologies applied to well annotated clinical cohorts, yielding a complex landscape of disease. The International Sarcoma Kindred Study was formed to investigate the heritable aspects of sarcoma. We have previously identified not only monogenic drivers but also polygenic contributions to sarcoma. Most recently we have completed whole genome sequencing (WGS) of 1700 sarcoma probands. A rare variant burden analysis and novel ontologic analysis identified two new pathways implicated in sarcoma risk. Heritable risk correlates with early age at diagnosis. The Genetic Cancer Risk in the Young Study is a cohort of 1100 young ( $0-40$ years) cancer patients. Using WGS we aim to investigate further the heritable drivers of disease. Translation of genetic insights into better management of risk is pivotal to the value proposition for genomics, most likely through early cancer detection. In 2012 we began a surveillance study utilising whole body MRI. There are currently 110 participants and we have detected 28 new primary malignancies that have been treated with curative intent. We aim to inform identification of those at greatest heritable risk and influence clinical risk management in the genomic era of personalised medicine.

\section{EXOMES IN PRENATAL TESTING: AN INTRODUCTION TO THE PREGEN STUDY}

Michael F. Buckley ${ }^{1}$, Tony Roscioli ${ }^{1,2}$, on behalf of the PreGen consortium investigators

${ }^{1}$ Randwick Genetics Laboratory, NSW Health Pathology, Randwick, NSW, Australia; and ${ }^{2}$ Neurosciences Research Australia, University of NSW, Randwick, NSW, Australia

Rapid turn-around time whole exome sequencing was developed initially to provide diagnoses for neonates in intensive care units, and has quickly become an accepted component of clinical genomics practice. The PreGen study was established in 2020 to investigate the clinical utility and cost effectiveness of establishing a genomic diagnosis in the second trimester of pregnancy for foetuses with ultrasound-detected structural malformations. This presentation will showcase two case studies of antenatal genomic diagnosis and place these results in the context of the current literature.

Acknowledgement: The funding support of the Genomic Health Futures Mission of the Australian Government is acknowledged with gratitude.

\section{EPIGENETIC CHANGES DURING TUMOUR DEVELOPMENT - FUNCTIONAL EPIGENETICS STUDY}

Clare Stirzaker $^{1,2}$, Ksenia Skvortsova ${ }^{1}$, Susan J. Clark ${ }^{1,2}$ ${ }^{1}$ Epigenetics Research Laboratory, Genomics and Epigenetics Division, Garvan Institute of Medical Research, Sydney, NSW, Australia; and ${ }^{2}$ St Vincent's Clinical School, UNSW Sydney, Sydney, NSW, Australia

DNA methylation plays a key role in demarcation of regulatory regions, including promoter-associated $\mathrm{CpG}$ islands. While $\mathrm{CpG}$ islands are typically maintained in an unmethylated state, a proportion of islands are subject to hypermethylation during tumorigenesis. A key question is to understand what triggers the aberrant changes to the DNA methylation landscape in cancer. Using methylome sequencing, we have identified a subset of distinct $\mathrm{CpG}$ islands that display partial DNA methylation 